\title{
Conservation Agriculture Based Practices Affect the Weed Dynamics in Spring Maize
}

\author{
Saugat Dahal $^{1, *}$, Tika Bahadur Karki ${ }^{2}$ \\ ${ }^{1}$ Tribhuwan University, Institute of Agriculture and Animal Science, Rampur, Chitwan, Nepal \\ ${ }^{2}$ Nepal Agricultural Research Council, National Maize Research Program, Rampur, Chitwan, Nepal \\ *Corresponding author: saugatdhl11@gmail.com
}

Received October 11, 2014; Revised November 12, 2014; Accepted December 28, 2014

\begin{abstract}
In order to find out the alternative methods for the weed management in spring maize (Zea mays L.) in Terai Nepal, a study was carried out in conservation agriculture under maize-rice cropping system in Rampur, Nepal during 2013. The weeds number and dry weight were compared in tillage methods viz. conventional and no tillage; residue management viz. residue kept and removed; fertilizer doses viz. research recommended doses and farmer dose; and weed management viz. herbicide use and manual weeding. No tillage, residue kept and atrazine applied @ $1.5 \mathrm{~kg} \mathrm{a.i} \mathrm{ha}^{-1}$ as pre-emergence had significantly lower number and lower dry weight of grasses and broadleaf weeds as compared to conventional tillage, residue removed and manual weeding at an interval of one month. While the number and dry weight of sedges was not significantly influenced by tillage, residue and fertilizer management. Manual weeding had significantly less number and weight of sedges over atrazine use. The total number and dry weight of weeds were less in no tillage, residue retention and atrazine use over the conventional tillage, residue removed and manual weeding. It is concluded that no tillage, residue retention and atrazine use can be the alternative technologies for weed management.
\end{abstract}

Keywords: weeds, tillage, residue, fertilizer, herbicide, number, dry weight

Cite This Article: Saugat Dahal, and Tika Bahadur Karki, “Conservation Agriculture Based Practices Affect the Weed Dynamics in Spring Maize.” World Journal of Agricultural Research, vol. 2, no. 6A (2014): 25-33. doi: 10.12691/wjar-2-6A-5.

\section{Introduction}

One of the major limiting factors for efficient crop production is the weed problem that ranges the yield loss in maize from $28-93 \%$ due to unchecked weed growth [1]. Weeds compete with crop for nutrients, moisture, light as well as carbon dioxide, so that it was recorded $40 \%$ of maize production was hampered worldwide due to competition with weeds [2]. Thus, weed is the major problem for loss potential of crop (37\%) as compared to other loss potential i.e. animal pest $18 \%$, fungal and bacterial pathogen $16 \%$, and virus $2 \%$ [3].

Conservation agriculture includes all form of crop management practices involving minimum soil disturbance, retention of residue on soil surface, use of herbicide for weed control and crop rotation [4]. The weed density is increased due to conventional tillage as compared to no tillage [5]. This is due to the weed seed bank below the soil is taken up where there is favorable place for germination of weed seeds. Though, there may be the limited literature about weed suppression due to mulching in tropical reason, crop residue has reduced the weed density and biomass in temperate regions [6]. Nitrogen application helps to develop competitive potential in crop by its vigorous growth while the herbicide use is economic and easy method of weed control [7]. Herbicidal method of weed control is one of the most usefulness and effective measure in maize [8]. Chemical weed control methods are much more economical than hand weeding in conservation agriculture [9].

Due to the frequent monsoon in rainy season, too much hot in spring season and acute shortage of labor, mechanical or hand weeding operation is nearly impossible at the required time. In this situation, alternative method for weed management is necessary to develop to minimize the effect of weeds for crop production. The cultivation and financing problem is hard to manage the weeds from field; many weed species are difficult to kill [10]. Weed communities in different places are influenced due to various factors [11]; as a result weed management can be applied in conservation agriculture as double profit basis. No tillage, residue retention and use of atrazine for long period affects the yield of maize [12] and along with this, the effect on weed number and weed dry biomass was observed due to conservation agriculture.

\section{Materials and Methods}

The field experiment was carried out in the farm of National Maize Research Program (NMRP), Chitwan, Nepal, at the elevation of 256 masl in the inner Terai region, from February to June 2013, during spring season. 
The cropping history was with Maize -Rice cropping till fifth season of maize cultivation. The research, having four factors with two level of each, was conducted in strip-split plot design. In strip, vertical factor and horizontal factor with tillage method and residue management respectively; in split, main plot factor and sub- plot factor with fertilizer and weed management practices respectively were applied. Tillage factor with i) conventional tillage and ii) no tillage; residue management with i) $35 \mathrm{~cm}$ rice residue retained and ii) residue removed; fertilizer management with i) research dose i.e. 180:115:160 Kg NPK ha ${ }^{-1}$ under yield target of $8 \mathrm{t} \mathrm{ha}^{-1}$ for hybrid maize [13] ii) farmer practice level of fertilizer dose i.e. 10 ton ha ${ }^{-1}$ FYM +70:30:50 kg NPK ha' ${ }^{-1}$ [14]; and weed management with i) herbicide use i.e. Atrazine, pre-emergence only @ 1.5 kg a. i. ha ${ }^{-1}$ within 48 hours of seeding and ii) manual weeding i.e. hand pulling i.e. at the interval of one month as not to disturb the soil under no tillage. Fertilizer was applied as i) nitrogen in three split dose i.e. basal dose, $\mathrm{V}_{6}$ stage and $\mathrm{V}_{10}$ stage of maize with system for the fourth season with no tillage, residue retention and atrazine use continuously in the same plot

equal amount ii) Phosphorus in basal dose only iii) Potassium in $50 \%$ basal dose and $50 \% \mathrm{~V}_{10}$ stage of maize. Hybrid maize (Rampur Hybrid- 2), within a single plot of $34.02 \mathrm{~m}^{2}(5.4 \mathrm{~m} \times 6.3 \mathrm{~m})$ was planted with spacing of 60 $\mathrm{cm} \times 30 \mathrm{~cm}$. At initial stage weeds were killed by using glyphosate@5ml/lit in all treatments fifteen days earlier to sowing before field preparation. Weed density and weed dry bio-mass was calculated to evaluate the weed infestation in maize field. Data were recorded before a day of manual weeding at interval of one month. For data analysis MSTAT-C package was used and for comparing the means Duncan's Multiple Range Test (DMRT) was used.

\subsection{Weather Condition during Experimentation}

The mean maximum and minimum temperature ranged from $25.73{ }^{\circ} \mathrm{C}$ to $34.98{ }^{\circ} \mathrm{C}$ and $7.02{ }^{\circ} \mathrm{C}$ to $26.33{ }^{\circ} \mathrm{C}$ respectively during the crop season. Rainfall was recorded about $1107.1 \mathrm{~mm}$ during entire growing season for maize (Figure 1).

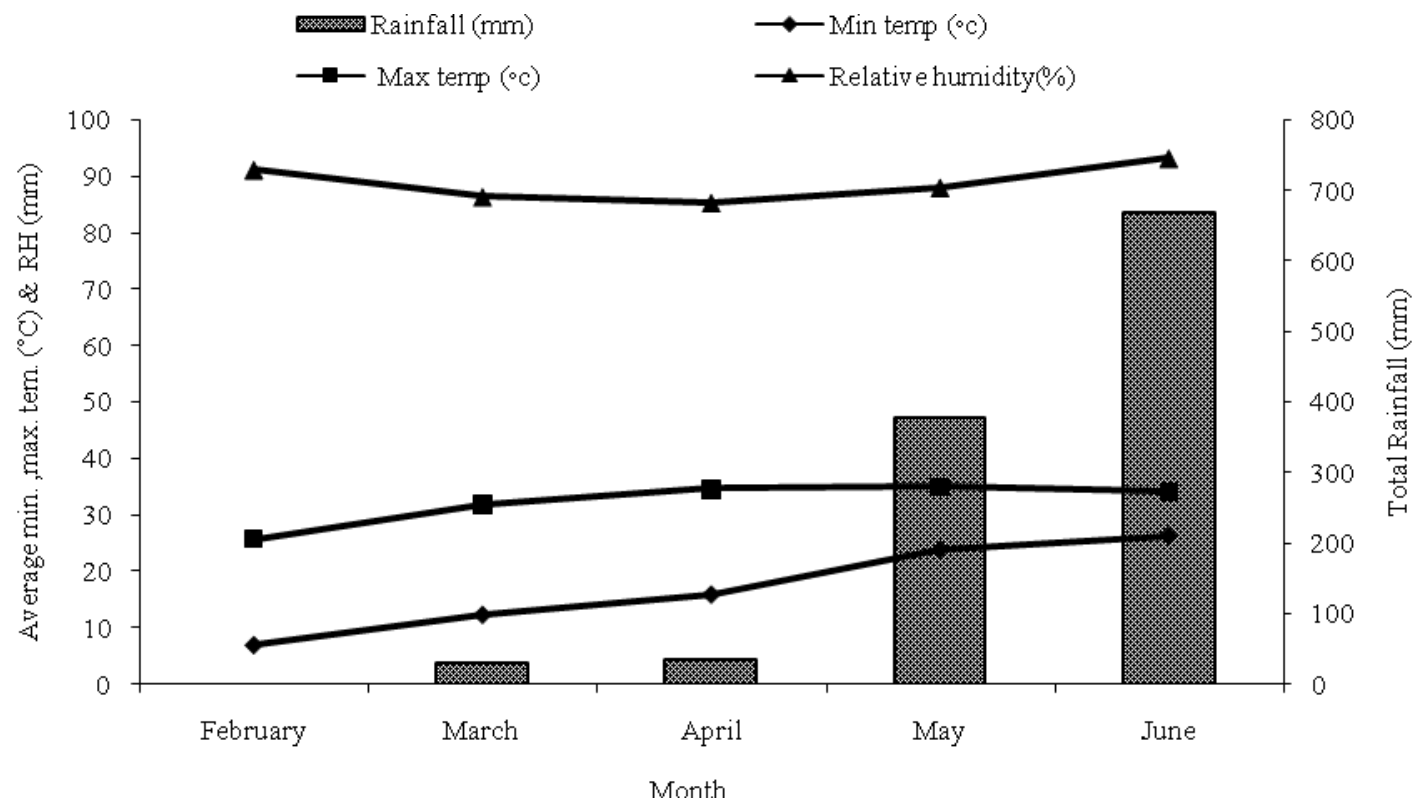

Figure 1. Weather condition during the course of experimentation at Rampur, Chitwan, Nepal during February to June, 2013

Table 1. Weed species composition in the field

\begin{tabular}{|c|c|c|c|c|}
\hline Scientific name & Common name & Family & Class & Habit \\
\hline \multicolumn{5}{|l|}{ Grasses } \\
\hline Cynodon dactylon (L.) Pers. & Bermuda grass & Poaceae & M & $\mathrm{PH}$ \\
\hline Digitaria ciliaris (Retz.) Koel. & Crab grass & Poaceaea & M & $\mathrm{AH}$ \\
\hline Eluesine indica (L.) Gaertn. & Goosegrass & Poaceae & M & $\mathrm{AH}$ \\
\hline Eleusine aegyptiaca (L.) & Crowfoot grass & Poaceae & M & $\mathrm{AH}$ \\
\hline Pennisetum glaucum (L.) R. Br & Pearlmillet & Poaceae & M & $\mathrm{AH}$ \\
\hline \multicolumn{5}{|l|}{ Sedges } \\
\hline Cyperus iria L. & Rice flat sedge & Сyperaceae & $\mathrm{M}$ & $\mathrm{AH}$ \\
\hline Cyperus rotundus L. & Purple nut sedge & Сyperaceae & M & $\mathrm{AH}$ \\
\hline \multicolumn{5}{|l|}{ Broad leaf weeds } \\
\hline Ageratum conyzoides L. & Goat weed & Compositae & $\mathrm{D}$ & $\mathrm{AH}$ \\
\hline Euphorbia hirta L. & Garden spurge & Euphorbiaceae & $\mathrm{D}$ & $\mathrm{AH}$ \\
\hline Humulus japonicas & Asian hop & Cannabaceae & $\mathrm{D}$ & $\mathrm{ACV}$ \\
\hline Commelina bengalensis Linn. & Day flower & Commelinaceae & M & $\mathrm{PH}$ \\
\hline
\end{tabular}




\section{Results and Discussion}

\subsection{Species Composition}

There were 12 major species of weeds in the field (Table 1). Among them Cynodon dactylon was the major in number and biomass.

\subsection{Number of Grasses}

Number of grasses was significantly influenced by tillage, residue, fertilizer and weed management factor. No tillage and residue retained level had significantly $(\mathrm{p} \leq 0.05)$ lower number of grasses as compared to conventional tillage and residue removed level respectively at 30, 60 and 90 days after sowing (DAS) of maize. In fertilizer factor, recommended dose of fertilizer had significantly lower number of grasses as compared to farmer practice level of fertilizer dose at 60 and 90 DAS of maize. In weed management factor, herbicide used level had significantly $(p \leq 0.05)$ lower number of grasses as compared to manual weeding at 30 DAS of maize but it was insignificant at 60 DAS and increased the number of weeds in herbicide used level than manual weeding at 90 DAS of maize.

Due to the sufficient moisture in long term no tillage treatment; there is sufficient growth of maize [15], which creates the shedding effect of maize for weed growth and germination. Similar effect was observed by [16] on spring maize. Long term use of rice and maize residue converts to mineralized nutrient which causes sufficient growth of maize [15], may be the probable reason for the suppression of weeds by the shedding effect. Similarly, in high dose of fertilizer also, there is sufficient growth of maize [15], which might have caused the suppression of weeds by the shedding effect. In weed management factor, there was significant effect of herbicide at 30 DAS of maize and it was just opposite at 90 DAS of maize due to weed free made at the interval of one month in manual weeding but increasing of weeds along with previous weeds in herbicide used level.

\subsection{Dry Weights of Grasses}

Dry weight of grasses was significantly influenced by tillage, residue, fertilizer and weed management factor. No tillage had significantly $(\mathrm{p} \leq 0.05)$ lower number of grasses as compared to conventional tillage at 30,60 and 90 DAS of maize. Residue retained level had also significantly lower dry weight of grasses as compared to residue removed level at 30, 60 and 90 DAS of maize. Research dose of fertilizer had significantly $(p \leq 0.01)$ lower dry weights of grasses as compared to farmer dose of fertilizer at 90 DAS of maize. In weed management factor, herbicide used level had significantly $(p \leq 0.01)$ lower dry weights of grasses as compared to manual weeding at 30 DAS of maize; insignificant at 60 DAS of maize and at 90 DAS of maize, herbicide used level had significantly $(p \leq 0.01)$ higher dry weight of narrow leaf weed as compared to manual weeding respectively.

There is similar effect in dry weight of grasses as number, except at 90 DAS of maize. It might be due to the growth of remaining weeds from initial time in herbicide used treatment.

\subsection{Number of Broadleaf Weeds}

Number of broadleaf weeds was significantly influenced by tillage residue and weed management factor whereas it was not significantly influenced by fertilizer management. No tillage and residue retained level had significantly lower number of broadleaf weeds as compared to conventional tillage and residue removed level respectively at 30, 60 and 90 DAS of maize. In weed management factor, herbicide used level had significantly $(\mathrm{p} \leq 0.01)$ lower number of weeds as compared to manual weeding at 30, 60 and 90 DAS of maize. In fertilizer factor, recommended dose of fertilizer had significantly lower weed number as compared to farmer dose of fertilizer.

There is similar effect in number of broadleaf weeds as the grasses due to tillage, residue and fertilizer. But in weed management factor, there is similar effect from 30 DAS to 90 DAS of maize, which might have caused due to more effect of atrazine to annual broadleaf weeds rather than grasses [17].

\subsection{Dry Weight of Broadleaf Weeds}

Dry weight of broadleaf weeds was significantly influenced by tillage, residue and weed management factor whereas it was not significantly influenced by fertilizer management. No tillage had significantly lower dry weight of broadleaf weeds as compared to conventional tillage at 30,60 and 90 DAS of maize. In residue management factor, residue retained level had significantly $(p \leq 0.05)$ lower dry weight of broadleaf weeds as compared to residue removed level at 30, 60 and 90 DAS of maize. In weed management factor, herbicide used level had significantly $(\mathrm{p} \leq 0.01)$ lower dry weight of broadleaf weeds as compared to manual weeding at 30, 60 and 90 DAS of maize. But in fertilizer management factor it was not significantly influenced and recommended dose of fertilizer had lower dry weight of broad leaf weeds as compared to farmer dose of fertilizer at 30, 60 and 90 DAS of maize.

\subsection{Number of Sedges}

Number of sedges was not significantly influenced by tillage, residue and fertilizer management whereas it was significantly influenced by weed management factor at 30 DAS of maize. At 30 DAS of maize, manual weeding had significantly $(\mathrm{p} \leq 0.01)$ lower weeds number as compared to herbicide used level. Though, other factors had not significant influence on sedges number but no tillage, residue removed level, recommended dose of fertilizer had lower number of sedges as compared to conventional tillage, residue retained and farmer dose of fertilizer respectively at 30, 60 and 90 DAS of maize.

The higher number of sedges in residue retained plot might be due to favorable condition of wet and moist area due to residue. It was also found that there is no effect of atrazine for sedges.

\subsection{Dry Weight of Sedges}

Dry weight of sedge was not significantly influenced by tillage, residue and fertilizer management whereas it was significantly influenced by weed management factor at 30, 60 and 90 DAS of maize. In weed management factor, 
manual weeding had significantly lower dry weight of sedges as compared to herbicide used level. Similar as number of sedges, no tillage, residue removed level, recommended dose of fertilizer had lower dry weight of sedges as compared to conventional tillage, residue retained and farmer dose of fertilizer respectively at 30, 60 and 90 DAS of maize.

\subsection{Total Number of Weeds}

Total number of weeds was significantly influenced by tillage, residue and weed management factor whereas it was not significantly influenced by fertilizer management factor. No tillage had significantly lower number of weeds as compared to conventional tillage at 30, 60 and 90 DAS of maize. In residue management factor, residue retained level had significantly lower number of weeds as compared to residue removed level at 60 and 90 DAS of maize. In weed management factor, herbicide used level had significantly lower number of weeds as compared to manual weeding at 30 and 60 DAS of maize. Although, fertilizer management factor had not significant influence but research dose of fertilizer had lower number of weeds as compared to farmer dose of fertilizer at 30, 60 and 90 DAS of maize.

The soil in no tillage is less disturbed to bring weed seed to the surface or buried in the soil. [18] also reported similar result in no tillage and conventional tillage for annual grass and annual broadleaf weeds. Crop residue prevents weed seed from germinating and growing due to less space and light and later producing seed from weed is prevented. Maize grown with high fertilizer grows vigorously and creates shedding effect to suppress the weeds before they grow. Similar result was obtained by [19], while using the fertilizer dose of 0:0 and 60:60 N:P $\mathrm{ha}^{-1}$. Atraizne as pre-emergence herbicide controls most of annual broadleaf weeds and some annual grasses. So that, there was no significant difference between herbicide used and manual weeding at later stage i.e. 90 DAS of maize due to increased number of grasses and sedges. [20], reported less weed density by using atrazine and glyphosate as pre-plant than hoe weeding at 3 and 7 week after planting of maize.

\subsection{Total Dry Weight of Weeds}

Total dry weight of weeds was significantly influenced by tillage, residue, fertilizer and weed management. No tillage had significantly $(\mathrm{p} \leq 0.01)$ lower dry weight of weeds as compared to conventional tillage and residue retained level had significantly $(\mathrm{p} \leq 0.05)$ lower dry weight of weeds as compared to residue removed level at 30, 60 and 90 DAS of maize. In fertilizer management factor, recommended dose of fertilizer had significantly $(\mathrm{p} \leq 0.01)$ lower dry weight of weeds as compared to farmer dose of fertilizer at 90 DAS of maize. In weed management factor, at 30 and 60 DAS of maize, herbicide used level had significantly $(p \leq 0.01)$ lower dry weight of weeds as compared to manual weeding while at 90 DAS of maize manual weeding had significantly $(\mathrm{p} \leq 0.01)$ lower dry weight of weeds as compared to herbicide used.

Table 2. Effect of tillage, residue, fertilizer and weed management on number and dry weight of weeds at 30 DAS of maize

\begin{tabular}{|c|c|c|c|c|c|c|c|c|}
\hline \multirow{3}{*}{ Treatments } & \multicolumn{8}{|c|}{ Types of weeds at 30 DAS of maize } \\
\hline & \multicolumn{4}{|c|}{ Number of weeds $\mathrm{m}^{-2}$} & \multicolumn{4}{|c|}{ Dry weight of weed $\mathrm{m}^{-2}$ (gm) } \\
\hline & Grasses & Broadleaf & Sedges & Total & Grasses & Broadleaf & Sedges & Total \\
\hline \multicolumn{9}{|c|}{ Tillage method } \\
\hline $\mathrm{CT}$ & $\begin{array}{l}7.75^{\mathrm{a}} \\
(63.71) \\
6.09^{\mathrm{b}} \\
(46.33)\end{array}$ & $\begin{array}{l}5.84^{\mathrm{a}} \\
(42.75) \\
3.17^{\mathrm{b}} \\
(14.75)\end{array}$ & $\begin{array}{l}2.44 \\
(8.83) \\
1.46 \\
(2.33) \\
\end{array}$ & $\begin{array}{l}16.03^{\mathrm{a}} \\
(115.2) \\
10.72^{\mathrm{b}} \\
(63.42)\end{array}$ & $\begin{array}{l}3.43^{\mathrm{a}} \\
(12.27) \\
2.34^{\mathrm{b}} \\
(5.83)\end{array}$ & $\begin{array}{l}3.04^{\mathrm{a}} \\
(11.3) \\
2.14^{\mathrm{b}} \\
(6.06)\end{array}$ & $\begin{array}{l}1.23 \\
(0.65) \\
1.06 \\
(0.14)\end{array}$ & $\begin{array}{l}7.70^{\mathrm{a}} \\
(24.25) \\
5.54^{\mathrm{b}} \\
(12.03)\end{array}$ \\
\hline LSD & $0.95^{*}$ & $1.75^{*}$ & NS & $1.42 * *$ & $0.72 *$ & $0.51^{* *}$ & NS & $2.13^{* *}$ \\
\hline SEm \pm & 0.156 & 0.288 & 0.312 & 0.101 & 0.118 & 0.037 & 0.071 & 0.152 \\
\hline \multicolumn{9}{|c|}{ Residue management } \\
\hline $\mathrm{RR}$ & $\begin{array}{l}5.55^{\mathrm{b}} \\
(36.75) \\
8.29^{\mathrm{a}} \\
(73.29) \\
\end{array}$ & $\begin{array}{l}3.76^{\mathrm{b}} \\
(22.58) \\
5.26^{\mathrm{a}} \\
(34.92) \\
\end{array}$ & $\begin{array}{l}2.32 \\
(8.83) \\
1.58 \\
(2.33) \\
\end{array}$ & $\begin{array}{l}11.63 \\
(68.17) \\
15.13 \\
(110.54) \\
\end{array}$ & $\begin{array}{l}2.30^{\mathrm{b}} \\
(5.41) \\
3.47^{\mathrm{a}} \\
(12.69) \\
\end{array}$ & $\begin{array}{l}2.09^{\mathrm{b}} \\
(5.66) \\
3.10^{\mathrm{a}} \\
(11.7) \\
\end{array}$ & $\begin{array}{l}1.20 \\
(0.59) \\
1.08 \\
(0.19) \\
\end{array}$ & $\begin{array}{l}5.59^{\mathrm{b}} \\
(11.66) \\
7.65^{\mathrm{a}} \\
(24.62) \\
\end{array}$ \\
\hline LSD & $2.71^{*}$ & $1.13^{*}$ & NS & NS & $1.11^{*}$ & $0.48^{*}$ & NS & $1.21 *$ \\
\hline $\operatorname{SEm} \pm$ & 0.444 & 0.186 & 0.549 & 0.868 & 0.182 & 0.079 & 0.096 & 0.198 \\
\hline \multicolumn{9}{|c|}{ Fertilizer doses } \\
\hline FD & $\begin{array}{l}7.08 \\
(56.00)\end{array}$ & $\begin{array}{l}4.61 \\
(34.50)\end{array}$ & $\begin{array}{l}2.14 \\
(5.83)\end{array}$ & $\begin{array}{l}13.82 \\
(96.33)\end{array}$ & $\begin{array}{l}2.83 \\
(8.33)\end{array}$ & $\begin{array}{l}2.63 \\
(9.72)\end{array}$ & $\begin{array}{l}1.16 \\
(0.43)\end{array}$ & $\begin{array}{l}6.62 \\
(18.47)\end{array}$ \\
\hline $\mathrm{RD}$ & $\begin{array}{l}6.76 \\
(54.04) \\
\end{array}$ & $\begin{array}{l}4.41 \\
(23.00)\end{array}$ & $\begin{array}{l}1.76 \\
(5.33) \\
\end{array}$ & $\begin{array}{l}12.93 \\
(82.38) \\
\end{array}$ & $\begin{array}{l}2.94 \\
(9.77) \\
\end{array}$ & $\begin{array}{l}2.56 \\
(7.69) \\
\end{array}$ & $\begin{array}{l}1.12 \\
(0.36) \\
\end{array}$ & $\begin{array}{l}6.62 \\
(17.81) \\
\end{array}$ \\
\hline LSD & NS & NS & NS & NS & NS & NS & NS & NS \\
\hline $\mathrm{SEm} \pm$ & 0.355 & 0.215 & 0.227 & 0.557 & 0.171 & 0.120 & 0.043 & 0.265 \\
\hline \multicolumn{9}{|c|}{ Weed management } \\
\hline Herbicide & $\begin{array}{l}5.13^{b} \\
(30.92)\end{array}$ & $\begin{array}{l}2.37^{\mathrm{b}} \\
(7.50)\end{array}$ & $\begin{array}{l}2.60^{\mathrm{a}} \\
(10.00)\end{array}$ & $\begin{array}{l}10.09^{b} \\
(48.42)\end{array}$ & $\begin{array}{l}2.14^{\mathrm{b}} \\
(4.61)\end{array}$ & $\begin{array}{l}1.40^{\mathrm{b}} \\
(1.16)\end{array}$ & $\begin{array}{l}1.25^{\mathrm{a}} \\
(0.70)\end{array}$ & $\begin{array}{l}4.79^{b} \\
(6.47)\end{array}$ \\
\hline Manual & $\begin{array}{l}8.72^{a} \\
(79.13)\end{array}$ & $\begin{array}{l}6.64^{a} \\
(50.00)\end{array}$ & $\begin{array}{l}1.30^{b} \\
(1.17)\end{array}$ & $\begin{array}{l}16.66^{a} \\
(130.29)\end{array}$ & $\begin{array}{l}3.63^{a} \\
(13.48)\end{array}$ & $\begin{array}{l}3.79^{a} \\
(16.2)\end{array}$ & $\begin{array}{l}1.04^{\mathrm{b}} \\
(0.09)\end{array}$ & $\begin{array}{l}8.46^{a} \\
(29.81) \\
\end{array}$ \\
\hline LSD & $0.84^{* *}$ & $1.13^{* *}$ & $1.13^{* *}$ & $1.11^{* *}$ & $0.46^{* *}$ & $0.43^{* *}$ & $0.20 * *$ & $0.51 * *$ \\
\hline SEm \pm & 0.204 & 0.273 & 0.274 & 0.268 & 0.111 & 0.105 & 0.047 & 0.123 \\
\hline $\mathrm{CV} \%$ & 14.45 & 29.62 & 68.93 & 9.80 & 18.80 & 19.87 & 20.28 & 9.10 \\
\hline Grand mean & $\begin{array}{l}6.92 \\
(55.02)\end{array}$ & $\begin{array}{l}4.51 \\
(28.75)\end{array}$ & $\begin{array}{l}1.95 \\
(5.58) \\
\end{array}$ & $\begin{array}{l}13.38 \\
(89.35)\end{array}$ & $\begin{array}{l}2.88 \\
(9.05) \\
\end{array}$ & $\begin{array}{l}2.59 \\
(8.70) \\
\end{array}$ & $\begin{array}{l}1.14 \\
(0.39) \\
\end{array}$ & $\begin{array}{l}6.62 \\
(18.14) \\
\end{array}$ \\
\hline
\end{tabular}

Data subjected to square-root $(\sqrt{\mathrm{X}+1})$ transformation; Figures in the parenthesis are original values; $*=$ significantly different at $\mathrm{p} \leq 0.05, * *=$

significantly different at $\mathrm{p} \leq 0.01$ by DMRT, LSD value differs according to the level of significance, $\mathrm{CT}=\mathrm{conventional}$ tillage, $\mathrm{NT}=$ no tillage, $\mathrm{RK}=$ residue kept, $\mathrm{RR}=$ residue removed, $\mathrm{FD}=$ farmer dose, $\mathrm{RD}=$ Research dose, $\mathrm{SFD}=\mathrm{Seed}$ fill duration, $\mathrm{DAS}=\mathrm{Days}$ after sowing. 
Table 3. Effect of tillage, residue, fertilizer and weed management on number and dry weight of weeds at 60 DAS of maize

\begin{tabular}{|c|c|c|c|c|c|c|c|c|}
\hline \multirow{3}{*}{ Treatments } & \multicolumn{8}{|c|}{ Types of weeds at 60 DAS of maize } \\
\hline & \multicolumn{4}{|c|}{ Number of weeds $\mathrm{m}^{-2}$} & \multicolumn{4}{|c|}{ Dry weight of weeds $\mathrm{m}^{-2}$ (gm) } \\
\hline & Grasses & Broadleaf & Sedges & Total & Grasses & Broadleaf & Sedges & Total \\
\hline \multicolumn{9}{|c|}{ Tillage methods } \\
\hline CT & $\begin{array}{l}8.71^{a} \\
(78.88)\end{array}$ & $\begin{array}{l}4.09^{a} \\
(27.00)\end{array}$ & $\begin{array}{l}3.68 \\
(20.00)\end{array}$ & $\begin{array}{l}16.48^{a} \\
(125.88)\end{array}$ & $\begin{array}{l}6.73^{a} \\
(49.04)\end{array}$ & $\begin{array}{l}2.98^{a} \\
(13.50)\end{array}$ & $\begin{array}{l}1.72 \\
(2.57)\end{array}$ & $\begin{array}{l}11.43^{\mathrm{a}} \\
(65.12)\end{array}$ \\
\hline NT & $\begin{array}{l}5.77^{b} \\
(38.42)\end{array}$ & $\begin{array}{l}2.84^{b} \\
(11.50)\end{array}$ & $\begin{array}{l}3.15 \\
(17.00)\end{array}$ & $\begin{array}{l}11.77^{\mathrm{b}} \\
(66.92)\end{array}$ & $\begin{array}{l}5.20^{b} \\
(32.59)\end{array}$ & $\begin{array}{l}1.92^{b} \\
(3.86)\end{array}$ & $\begin{array}{l}1.44 \\
(1.58)\end{array}$ & $\begin{array}{l}8.57^{b} \\
(38.04)\end{array}$ \\
\hline LSD & $2.92 *$ & $1.08 * *$ & NS & $2.39 *$ & $1.20 *$ & $0.82 *$ & NS & $1.23^{* *}$ \\
\hline $\mathrm{SEm} \pm$ & 0.480 & 0.077 & 0.126 & 0.393 & 0.198 & 0.135 & 0.073 & 0.088 \\
\hline \multicolumn{9}{|c|}{ Residue management } \\
\hline RK & $\begin{array}{l}5.94^{\mathrm{b}} \\
(41.46)\end{array}$ & $\begin{array}{l}3.21^{\mathrm{b}} \\
(15.00)\end{array}$ & $\begin{array}{l}3.49 \\
(19.50)\end{array}$ & $\begin{array}{l}12.64^{\mathrm{b}} \\
(75.96)\end{array}$ & $\begin{array}{l}4.84^{\mathrm{b}} \\
(27.77)\end{array}$ & $\begin{array}{l}2.04^{\mathrm{b}} \\
(5.00)\end{array}$ & $\begin{array}{l}1.63 \\
(2.28)\end{array}$ & $\begin{array}{l}8.52^{b} \\
(35.04)\end{array}$ \\
\hline $\mathrm{RR}$ & $\begin{array}{l}8.54^{a} \\
(75.83)\end{array}$ & $\begin{array}{l}3.73^{a} \\
(23.50)\end{array}$ & $\begin{array}{l}3.33 \\
(17.50)\end{array}$ & $\begin{array}{l}15.60^{a} \\
(116.83)\end{array}$ & $\begin{array}{l}7.09^{a} \\
(53.87)\end{array}$ & $\begin{array}{l}2.86^{a} \\
(12.36)\end{array}$ & $\begin{array}{l}1.53 \\
(1.88) \\
\end{array}$ & $\begin{array}{l}11.48^{a} \\
(68.11) \\
\end{array}$ \\
\hline LSD & $1.63^{*}$ & $0.37 *$ & NS & $1.61 * *$ & $1.50^{*}$ & $0.56^{*}$ & NS & $1.33^{*}$ \\
\hline SEm \pm & 0.268 & 0.061 & 0.417 & 0.115 & 0.247 & 0.092 & 0.156 & 0.219 \\
\hline \multicolumn{9}{|c|}{ Fertilizer doses } \\
\hline FD & $\begin{array}{l}7.72 \\
(64.17)\end{array}$ & $\begin{array}{l}3.79 \\
(22.08)\end{array}$ & $\begin{array}{l}3.47 \\
(17.67)\end{array}$ & $\begin{array}{l}14.98 \\
(103.92)\end{array}$ & $\begin{array}{l}6.20 \\
(42.29)\end{array}$ & $\begin{array}{l}2.69 \\
(10.68)\end{array}$ & $\begin{array}{l}1.63 \\
(2.13)\end{array}$ & $\begin{array}{l}10.51 \\
(55.10)\end{array}$ \\
\hline $\mathrm{RD}$ & $\begin{array}{l}6.76 \\
(53.13)\end{array}$ & $\begin{array}{l}3.15 \\
(16.42)\end{array}$ & $\begin{array}{l}3.35 \\
(19.33)\end{array}$ & $\begin{array}{l}13.26 \\
(88.88) \\
\end{array}$ & $\begin{array}{l}5.74 \\
(39.34)\end{array}$ & $\begin{array}{l}2.21 \\
(6.69)\end{array}$ & $\begin{array}{l}1.54 \\
(2.01)\end{array}$ & $\begin{array}{l}9.48 \\
(48.05) \\
\end{array}$ \\
\hline LSD & $0.88 *$ & NS & NS & NS & NS & NS & NS & NS \\
\hline SEm \pm & 0.269 & 0.256 & 0.453 & 0.691 & 0.500 & 0.158 & 0.133 & 0.573 \\
\hline \multicolumn{9}{|c|}{ Weed management } \\
\hline Herbicide & $\begin{array}{l}6.89 \\
(55.42)\end{array}$ & $\begin{array}{l}1.32^{\mathrm{b}} \\
(2.17)\end{array}$ & $\begin{array}{l}4.29 \\
(26.17)\end{array}$ & $\begin{array}{l}12.50^{\mathrm{b}} \\
(83.75)\end{array}$ & $\begin{array}{l}5.45 \\
(34.75)\end{array}$ & $\begin{array}{l}1.22^{\mathrm{b}} \\
(1.54)\end{array}$ & $\begin{array}{l}1.93^{\mathrm{a}} \\
(3.45)\end{array}$ & $\begin{array}{l}8.60^{\mathrm{b}} \\
(39.74)\end{array}$ \\
\hline Mannual & $\begin{array}{l}7.59 \\
(61.88) \\
\end{array}$ & $\begin{array}{l}5.61^{a} \\
(36.33) \\
\end{array}$ & $\begin{array}{l}2.54 \\
(10.83) \\
\end{array}$ & $\begin{array}{l}15.75^{\mathrm{a}} \\
(109.04)\end{array}$ & $\begin{array}{l}6.48 \\
(46.89) \\
\end{array}$ & $\begin{array}{l}3.68^{a} \\
(15.82) \\
\end{array}$ & $\begin{array}{l}1.23^{\mathrm{b}} \\
(0.70) \\
\end{array}$ & $\begin{array}{l}11.39^{\mathrm{a}} \\
(63.41) \\
\end{array}$ \\
\hline LSD & NS & $1.26^{* *}$ & NS & $3.03 * *$ & NS & $0.82 * *$ & $0.69 * *$ & $1.53^{* *}$ \\
\hline $\mathrm{SEm} \pm$ & 0.327 & 0.304 & 0.732 & 0.732 & 0.372 & 0.199 & 0.167 & 0.370 \\
\hline $\mathrm{CV} \%$ & 22.14 & 42.96 & 105.03 & 25.40 & 30.52 & 39.75 & 51.71 & 18.13 \\
\hline Grand mean & $\begin{array}{l}7.24 \\
(58.65)\end{array}$ & $\begin{array}{l}3.47 \\
(19.25)\end{array}$ & $\begin{array}{l}3.41 \\
(18.50)\end{array}$ & $\begin{array}{l}14.12 \\
(96.40)\end{array}$ & $\begin{array}{l}5.97 \\
(40.82)\end{array}$ & $\begin{array}{l}2.45 \\
(8.68)\end{array}$ & $\begin{array}{l}1.58 \\
(2.07)\end{array}$ & $\begin{array}{l}10.00 \\
(51.58)\end{array}$ \\
\hline
\end{tabular}

Data subjected to square-root $(\sqrt{\mathrm{X}+1})$ transformation; Figures in the parenthesis are original values; $*=$ significantly different at $\mathrm{p} \leq 0.05, * *=$ significantly different at $\mathrm{p} \leq 0.01$ by $\mathrm{DMRT}, \mathrm{LSD}$ value differs according to the level of significance, $\mathrm{CT}=$ conventional tillage, $\mathrm{NT}=$ no tillage, $\mathrm{RK}=$
residue kept, $\mathrm{RR}=$ residue removed, $\mathrm{FD}=$ farmer dose, $\mathrm{RD}=$ Research dose, $\mathrm{SFD}=$ Seed fill duration, $\mathrm{DAS}=\mathrm{Days}$ after sowing

Table 4. Effect of tillage, residue, fertilizer and weed management on number and dry weight of weeds at 90 DAS of maize

\begin{tabular}{|c|c|c|c|c|c|c|c|c|}
\hline \multirow{3}{*}{ Treatments } & \multicolumn{8}{|c|}{ Types of weeds at 90 DAS of maize } \\
\hline & \multicolumn{4}{|c|}{ Number of weeds $\mathrm{m}^{-2}$} & \multicolumn{4}{|c|}{ Dry weight of weed $\mathrm{m}^{-2}$ (gm) } \\
\hline & Grasses & Broadleaf & Sedges & Total & Grasses & Broadleaf & Sedges & Total \\
\hline \multicolumn{9}{|c|}{ Tillage methods } \\
\hline CT & $\begin{array}{l}8.15^{\mathrm{a}} \\
(68.83)\end{array}$ & $\begin{array}{l}4.78^{\mathrm{a}} \\
(27.75)\end{array}$ & $\begin{array}{l}3.68 \\
(20.00)\end{array}$ & $\begin{array}{l}16.61^{\mathrm{a}} \\
(116.58)\end{array}$ & $\begin{array}{l}7.87^{\mathrm{a}} \\
(73.03)\end{array}$ & $\begin{array}{l}2.37^{\mathrm{a}} \\
(6.56)\end{array}$ & $\begin{array}{l}1.72 \\
(2.57)\end{array}$ & $\begin{array}{l}11.96^{\mathrm{a}} \\
(82.15)\end{array}$ \\
\hline NT & $\begin{array}{l}6.26^{b} \\
(42.33)\end{array}$ & $\begin{array}{l}2.70^{b} \\
(12.58)\end{array}$ & $\begin{array}{l}3.16 \\
(17.00)\end{array}$ & $\begin{array}{l}12.12^{b} \\
(71.92)\end{array}$ & $\begin{array}{l}5.41^{\mathrm{b}} \\
(39.22)\end{array}$ & $\begin{array}{l}1.30^{\mathrm{b}} \\
(1.04)\end{array}$ & $\begin{array}{l}1.44 \\
(1.58)\end{array}$ & $\begin{array}{l}8.15^{\mathrm{b}} \\
(41.84)\end{array}$ \\
\hline LSD & $1.75^{*}$ & $1.16^{*}$ & NS & $2.80^{*}$ & $1.68 *$ & $0.43^{* *}$ & NS & $2.79 * *$ \\
\hline SEm \pm & 0.288 & 0.190 & 0.109 & 0.460 & 0.275 & 0.031 & 0.085 & 0.199 \\
\hline \multicolumn{9}{|c|}{ Residue management } \\
\hline RK & $\begin{array}{l}6.38^{\mathrm{b}} \\
(44.00)\end{array}$ & $\begin{array}{l}2.47^{b} \\
(10.08)\end{array}$ & $\begin{array}{l}3.50 \\
(19.50)\end{array}$ & $\begin{array}{l}12.34^{b} \\
(73.58)\end{array}$ & $\begin{array}{l}5.68^{b} \\
(41.34)\end{array}$ & $\begin{array}{l}1.32^{b} \\
(1.02)\end{array}$ & $\begin{array}{l}1.63 \\
(2.28)\end{array}$ & $\begin{array}{l}8.64^{b} \\
(44.64)\end{array}$ \\
\hline $\mathrm{RR}$ & $\begin{array}{l}8.04^{a} \\
(67.17)\end{array}$ & $\begin{array}{l}5.00^{a} \\
(30.25)\end{array}$ & $\begin{array}{l}3.33 \\
(17.50)\end{array}$ & $\begin{array}{l}16.38^{a} \\
(114.92)\end{array}$ & $\begin{array}{l}7.59^{a} \\
(70.90)\end{array}$ & $\begin{array}{l}2.35^{a} \\
(6.58)\end{array}$ & $\begin{array}{l}1.53 \\
(1.88)\end{array}$ & $\begin{array}{l}11.48^{a} \\
(79.35)\end{array}$ \\
\hline LSD & $0.84 *$ & $1.85 * *$ & NS & $2.74 *$ & $1.49 * *$ & $0.64 *$ & NS & $1.21 *$ \\
\hline SEm \pm & 0.138 & 0.132 & 0.367 & 0.450 & 0.106 & 0.105 & 0.146 & 0.202 \\
\hline \multicolumn{9}{|c|}{ Fertilizer doses } \\
\hline FD & $\begin{array}{l}7.83^{\mathrm{a}} \\
(66.00)\end{array}$ & $\begin{array}{l}3.93 \\
(23.33)\end{array}$ & $\begin{array}{l}3.47 \\
(17.67)\end{array}$ & $\begin{array}{l}15.24 \\
(107.00)\end{array}$ & $\begin{array}{l}7.26^{\mathrm{a}} \\
(65.42)\end{array}$ & $\begin{array}{l}1.95 \\
(4.13)\end{array}$ & $\begin{array}{l}1.62 \\
(2.13)\end{array}$ & $\begin{array}{l}10.84^{\mathrm{a}} \\
(71.69)\end{array}$ \\
\hline $\mathrm{RD}$ & $\begin{array}{l}6.58^{b} \\
(45.17)\end{array}$ & $\begin{array}{l}3.54 \\
(17.00) \\
\end{array}$ & $\begin{array}{l}3.36 \\
(19.33) \\
\end{array}$ & $\begin{array}{l}13.48 \\
(81.50) \\
\end{array}$ & $\begin{array}{l}6.02^{\mathrm{b}} \\
(46.83)\end{array}$ & $\begin{array}{l}1.72 \\
(3.46) \\
\end{array}$ & $\begin{array}{l}1.54 \\
(2.02) \\
\end{array}$ & $\begin{array}{l}9.28^{\mathrm{b}} \\
(52.31) \\
\end{array}$ \\
\hline LSD & $0.98 * *$ & NS & NS & NS & $0.92^{* *}$ & NS & NS & $1.11^{* *}$ \\
\hline SEm \pm & 0.207 & 0.165 & 0.448 & 0.583 & 0.194 & 0.127 & 0.125 & 0.234 \\
\hline \multicolumn{9}{|c|}{ Weed management } \\
\hline Herbicide & $\begin{array}{l}7.61 \\
(60.17)\end{array}$ & $\begin{array}{l}2.10^{b} \\
(5.17)\end{array}$ & $\begin{array}{l}4.29 \\
(26.17)\end{array}$ & $\begin{array}{l}14.00 \\
(91.50)\end{array}$ & $\begin{array}{l}9.65^{\mathrm{a}} \\
(97.78)\end{array}$ & $\begin{array}{l}1.27^{b} \\
(0.81)\end{array}$ & $\begin{array}{l}1.93^{\mathrm{a}} \\
(3.45)\end{array}$ & $\begin{array}{l}12.86^{\mathrm{a}} \\
(102.04)\end{array}$ \\
\hline Manual & $\begin{array}{l}6.81 \\
(51.00)\end{array}$ & $\begin{array}{l}5.37^{\mathrm{a}} \\
(35.17)\end{array}$ & $\begin{array}{l}2.54 \\
(10.83)\end{array}$ & $\begin{array}{l}14.72 \\
(97.00)\end{array}$ & $\begin{array}{l}3.62^{b} \\
(14.46)\end{array}$ & $\begin{array}{l}2.41^{\mathrm{a}} \\
(6.79)\end{array}$ & $\begin{array}{l}1.23^{\mathrm{b}} \\
(0.70)\end{array}$ & $\begin{array}{l}7.26^{\mathrm{b}} \\
(21.95)\end{array}$ \\
\hline LSD & NS & $0.70^{* *}$ & NS & NS & $0.83^{* *}$ & $0.49 * *$ & $0.51^{*}$ & $1.06^{* *}$ \\
\hline SEm \pm & 0.310 & 0.170 & 0.730 & 0.784 & 0.200 & 0.12 & 0.170 & 0.257 \\
\hline $\mathrm{CV} \%$ & 21.10 & 22.25 & 104.59 & 26.76 & 14.75 & 31.60 & 52.75 & 12.50 \\
\hline Grand mean & $\begin{array}{l}7.21 \\
(55.58)\end{array}$ & $\begin{array}{l}3.74 \\
(20.17)\end{array}$ & $\begin{array}{l}3.42 \\
(18.50)\end{array}$ & $\begin{array}{l}14.36 \\
(94.25)\end{array}$ & $\begin{array}{l}6.64 \\
(56.12)\end{array}$ & $\begin{array}{l}1.84 \\
(3.80)\end{array}$ & $\begin{array}{l}1.58 \\
(2.08)\end{array}$ & $\begin{array}{l}10.06 \\
(62.00)\end{array}$ \\
\hline
\end{tabular}


Due to the less soil disturbance, no tillage had less number of weed seeds in $0-15 \mathrm{~cm}$ soil in weedy plots [21]. The suppression of weed biomass due to the rice straw is probably due to little transmission or no transmission of solar radiation through residue [22]. Similar result was obtained by [23] in quality protein maize (QPM) due to rice straw. [24] reported that atrazine $(0.75 \mathrm{~kg} / \mathrm{ha})$ as preemergence and then followed by 2, 4-D (1 kg/ha) as postemergence at 30 DAS recorded significant less seed bank of weed over mechanical weeding. [20], reported less weed biomass by using atrazine and glyphosate as preplant than hoe weeding at 3 and 7 week after planting of maize. The total dry weight of weeds in herbicide used level is more in later stage i.e. 90 DAS of maize even having similar number; might be due to the remaining weeds from the initial stage of maize with higher weight in later stage because in herbicide used treatment weeds were not manually uprooted.

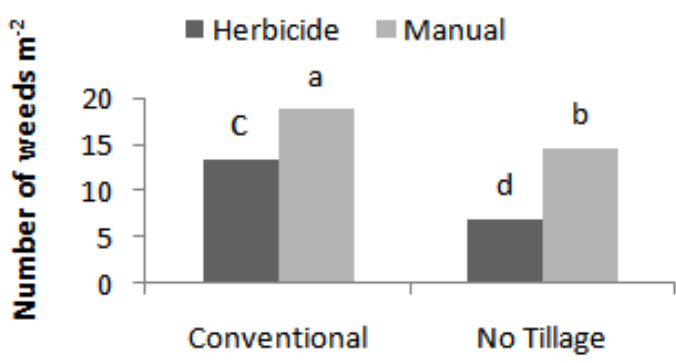

Tillage method

$2 a$

\subsection{Interaction Effect on Total Weed Number and Dry Weight}

Interaction effects on weed number was observed between tillage and weed management; residue and weed management; fertilizer and weed management; tillage, fertilizer and weed management; tillage, residue, fertilizer and weed management at 30 DAS of maize. At 90 DAS of maize, it was observed between tillage and residue; tillage, residue and weed management; tillage, residue and fertilizer management. Interaction effect on dry weight of weeds was observed between residue and weed management; fertilizer and weed management; tillage, residue and weed management; tillage, fertilizer and weed management at 30 DAS. At 60 DAS of maize, it was observed between tillage, fertilize and weed management; residue, fertilizer and weed management (Figure 2-Figure 9).

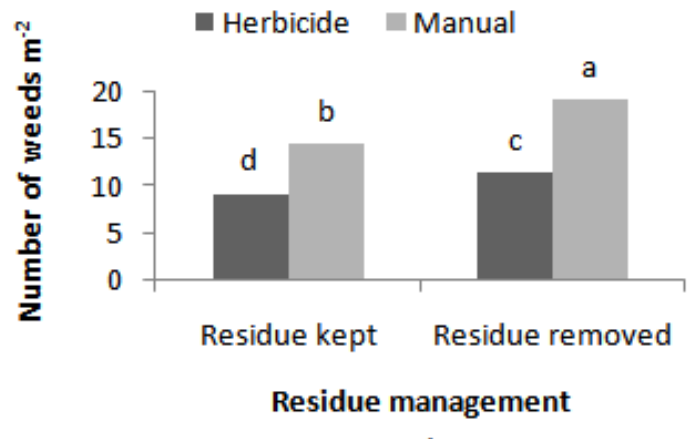

$2 \mathrm{~b}$

Figure 2. Interaction effect on number of weeds $\mathrm{m}^{-2}$ between (a) tillage and weed management $(\mathrm{p} \leq 0.05)$ (b) residue and weed management ( $\left.\mathrm{p} \leq 0.01\right)$ at 30 DAS of maize

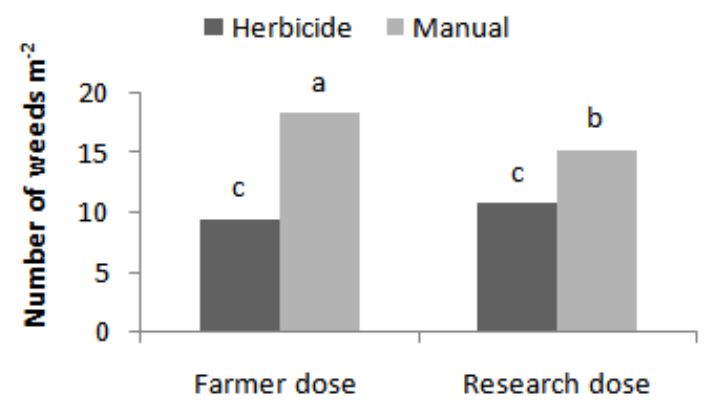

Fertilizer management

$3 a$

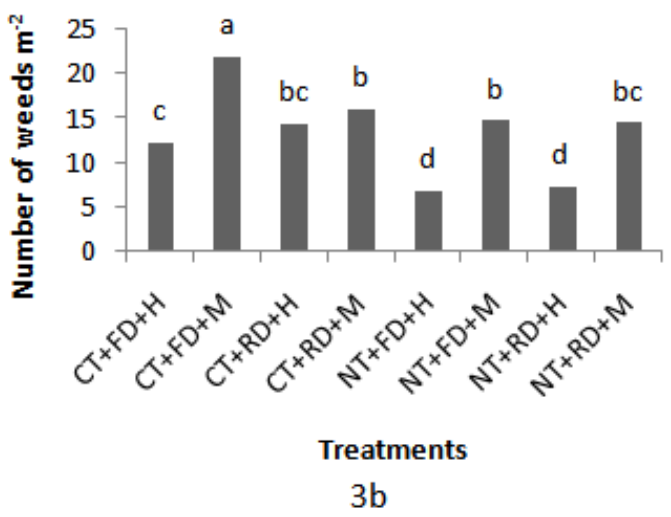

$3 b$

Figure 3. Interaction effect on number of weeds $\mathrm{m}^{-2}$ between (a) fertilizer and weed management ( $\mathrm{p} \leq 0.01$ ) (b) tillage, fertilizer and weed management $(\mathrm{p} \leq 0.01)$ at 30 DAS of maize

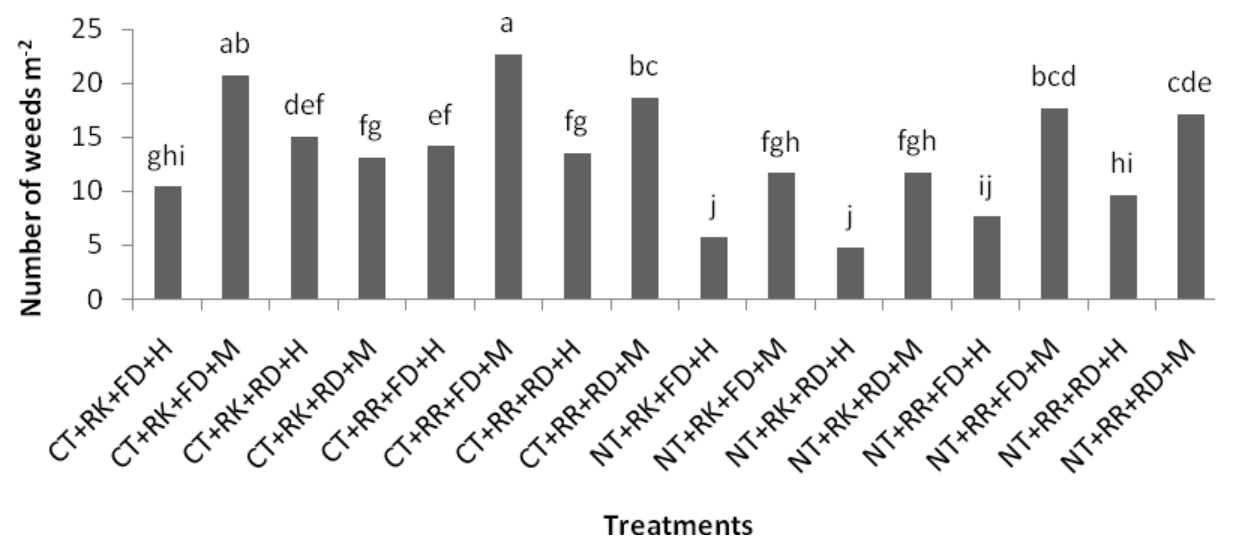

Figure 4. Interaction effect on number of weeds $\mathrm{m}^{-2}$ between tillage, residue, fertilizer and weed management ( $\left.\mathrm{p} \leq 0.01\right)$ at $30 \mathrm{DAS}$ of maize 

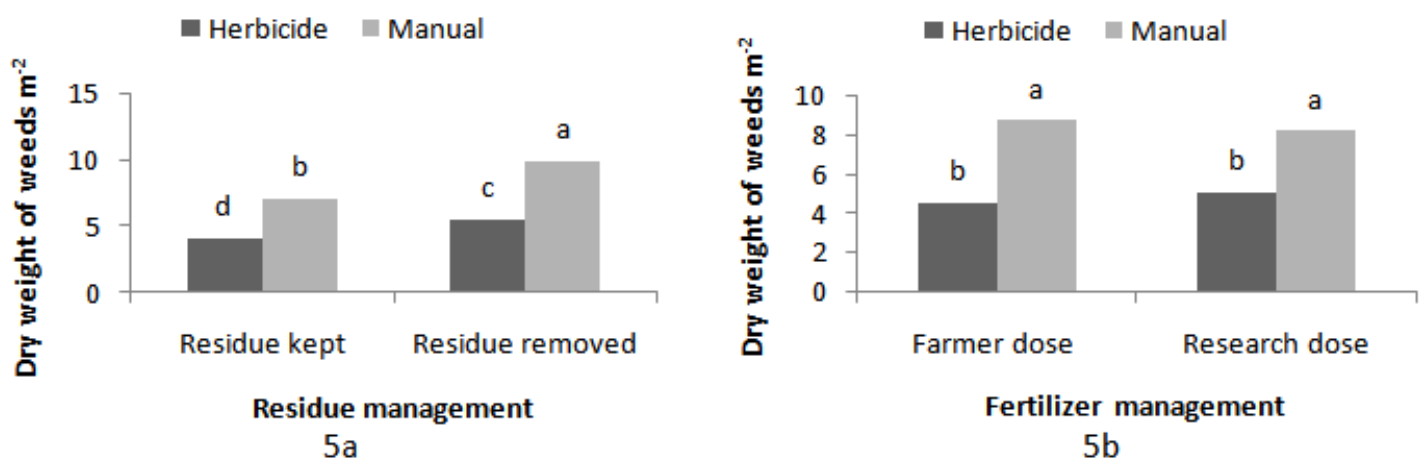

Figure 5. Interaction effect on dry weight of weeds $\mathrm{m}^{-2}$ between (a) residue and weed management ( $\mathrm{p} \leq 0.01$ ) (b) fertilizer and weed management $(\mathrm{p} \leq 0.01)$ at 30 DAS of maize
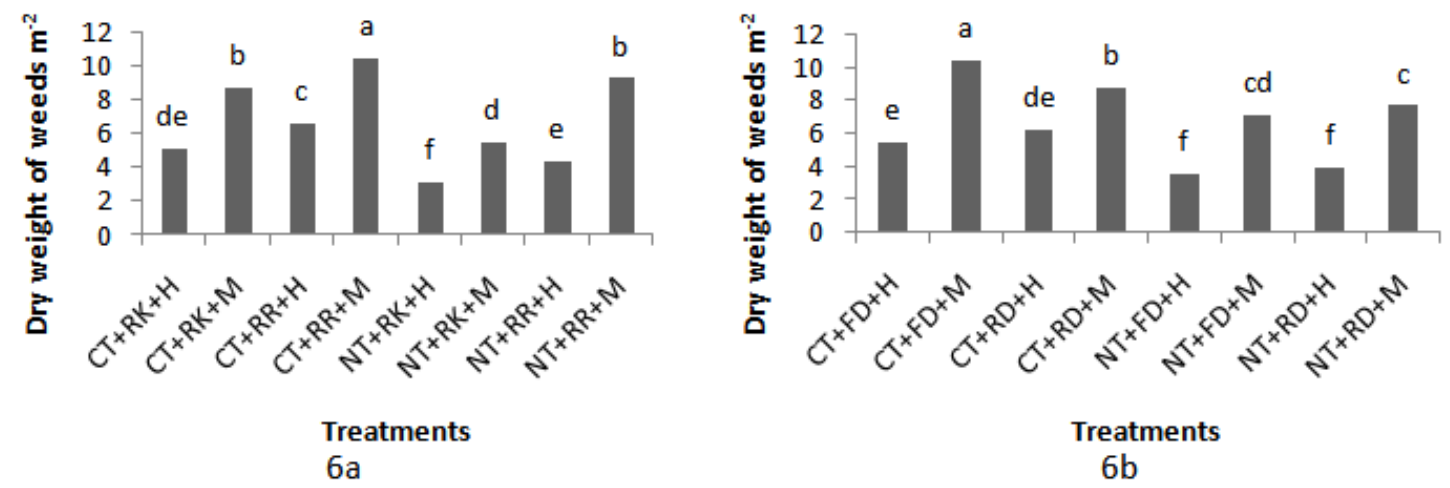

Figure 6. Interaction effect on dry weight of weeds $\mathrm{m}^{-2}$ between (a) tillage, residue and weed management ( $\left.\mathrm{p} \leq 0.01\right)$ (b) tillage, fertilizer and weed management $(\mathrm{p} \leq 0.01)$ at 30 DAS of maize
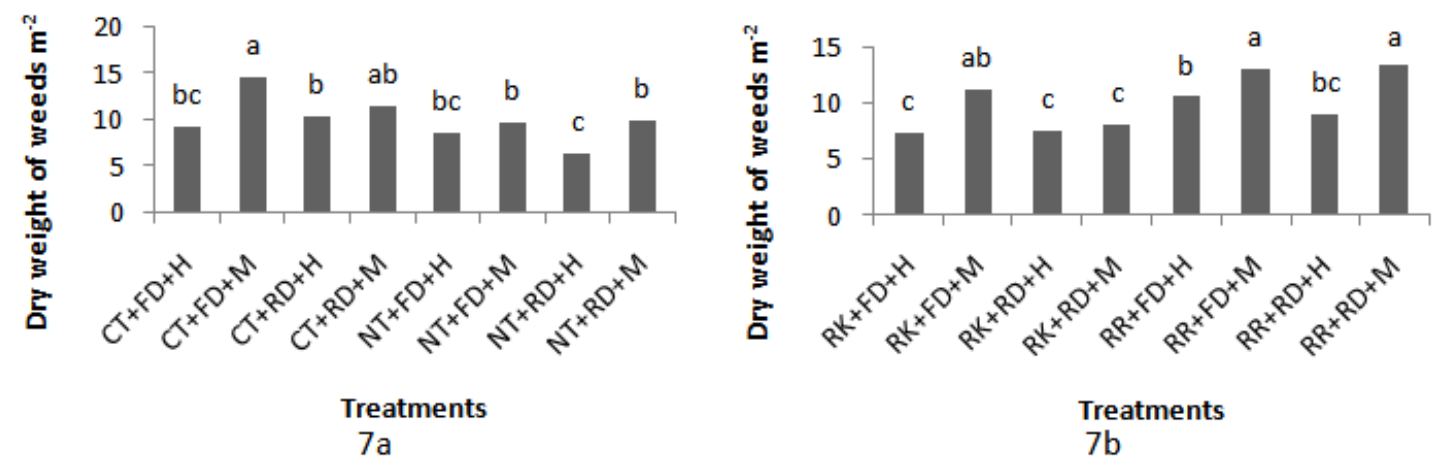

Figure 7. Interaction effect on dry weight of weeds $\mathrm{m}^{-2}$ between (a) tillage, fertilizer and weed management ( $\mathrm{p} \leq 0.01$ ) (b) residue, fertilizer and weed management $(\mathrm{p} \leq 0.05)$ at 60 DAS of maize

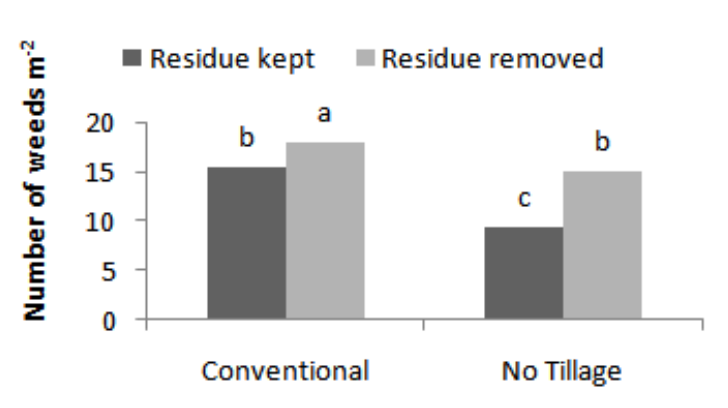

Tillage methods

$8 \mathrm{a}$

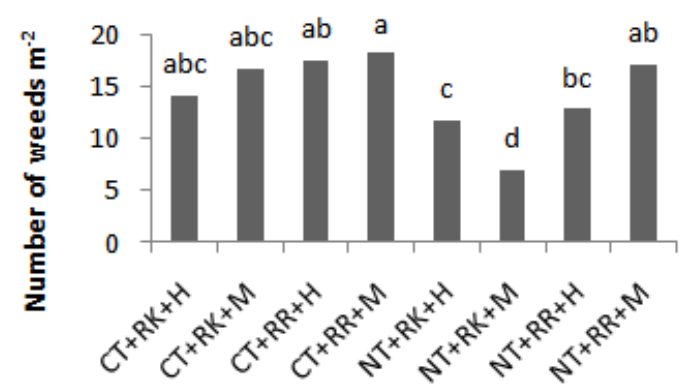

Treatments

$8 \mathrm{~b}$

Figure 8. Interaction effect on number of weeds $\mathrm{m}^{-2}$ between (a) tillage and residue management ( $\left.\mathrm{p} \leq 0.05\right)$ (b) tillage, residue and weed management $(\mathrm{p} \leq 0.05)$ at 90 DAS of maize

The weed number and dry weight of weeds was found less in no tillage, residue kept, research dose of fertilizer, herbicide use and the interaction between them. Interaction effect was observed more at 30 DAS of maize and less at 60 and 90 DAS of maize. During early phases of growth, weed competition is very sensitive to maize [25]. Maize has negligible effect on yield by the weeds after 4 weeks [26]. 


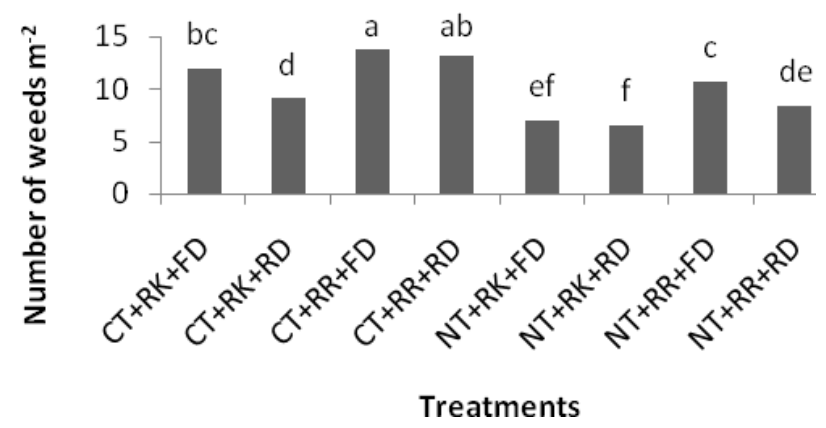

Figure 9. Interaction effect on number of weeds $\mathrm{m}^{-2}$ between tillage, residue and fertilizer management at 90 DAS of maize Broadleaf $\square$ Grasses $\square$ Sedges

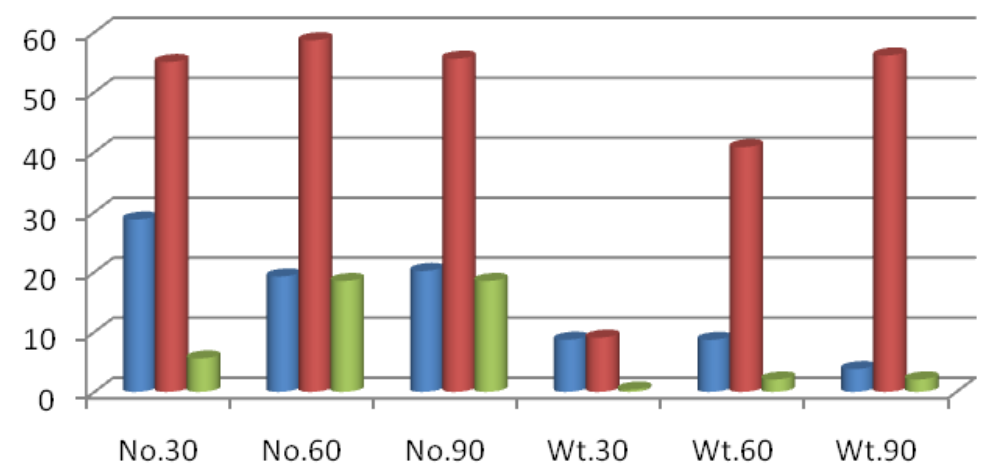

Figure 10. Effect of treatments in total number and total dry weight of weeds with time

The grasses number is increased within 60 DAS of maize and later slightly decreased at 90 DAS of maize but dry weight is increased in fast rate. Broadleaf weeds have decreasing rate in number and weight while sedges number and weight both are increased with time. Hence there is no shedding effect of maize for grasses and sedges (Figure 10).

\section{Conclusion}

No tillage, residue retention (35 $\mathrm{cm}$ rice residue retention), recommended doses of fertilizer (180:115:160 kg NPK ha ${ }^{-1}$ ) and application of atrazine @ $1.5 \mathrm{~kg} \mathrm{a.i.} \mathrm{ha}^{-1}$ within 48 hours of maize seeding) in rice-maize cropping system reduced significantly the weed population and biomass, hence could be an alternative method for weed management in Terai region of Nepal. The experiment need to be further verified in farmers' participatory research villages of similar ecologies.

\section{Acknowledgement}

Since, the financial support to the work was due to National Agriculture Research and Development Fund (NARDF), Nepal. The entire family of National Maize Research Program, Rampur, Chitwan, is highly acknowledged for their every support rendered to accomplish the field research successfully.

\section{References}

[1] Patel, V.J, Upadhyay, P.N, Patel, J.B. and Meisuriya, M.I, "Effect of herbicide mixture on weeds in kharif maize (Zea mays L.) under middle Gujrat conditions”. Indian journal of Weed science. 38 (1 and 2): 54-57. 2006.

[2] Oerke, E.C. and Dehne, H.W, "Safeguarding Production losses in major crops and the role of crop production”. Crop Prot. 23: 275285. 2004.

[3] Oerke, E.C, “Crop losses to pest”. J. Agri. Sci. 143:1-13. 2005.

[4] Harrington, L. and Erestein, O, "Conservation agriculture and resource conserving technologies- A global perpective”. In: Abrol, I.P, Gupta, R.K. and Malik, R.K, (ed.) Conservation agriculture status and prospects. New Delhi. pp 1-12. 2005.

[5] Shrestha, A, Knezevic, S.R, Roy, R.C, Ball-Coehlo, B.R. and Swanton, C.J, "Effect of tillage, cover crop and crop rotation on the composition of weed flora in a sandy soil”. Weed Res. 42: 7684. 2002.

[6] Bilalis, D, Sidiras, N, Economuu, G. and Vakali C, "Effect of different levels of wheat straw soil surface coverage on weed flora in Vicia faba crops”. J. Agron. Crop Sci. 189: 233-241. 2003.

[7] Khan, N.W, Khan, N. and Khan, J.A, "Interation of nitrogen fertilizer and herbicides for efficient weed management in maize crop”. Sarad J. Agric. 28(3): 457-463. 2012.

[8] Hassan, G, Tanveer, S, Khan, N. and Munir, M, "Integrating cultivars with reduced herbicides rates for weed management in maize”. Pak. J. Bot. 42 (3): 1923-1929. 2010.

[9] Tatenda, R.J. and Stanford, M, "Efficiency and economics of manual and chemical weed control strategies in the first year of conservation agriculture adoption in the highland areas of Zimbabwe”. Global Advanced Research Journal of Agricultural Science 2(9): 231-241. 2013.

[10] Farkas, A, "Soil management and tillage possibilities in weed control”. Herbologia 7(1): 9-23. 2006.

[11] Cardina, J, Herms, C.P. and Doohan D.J, "Crop rotation and tillage system effects on weed seedbanks”. Weed Science. 50: 448460. 2002.

[12] Dahal, S, Karki, T.B, Amgain, L.P. and Bhattachan, B.K, "Tillage, residue, fertilizer and weed management on phenology and yield of spring maize in Terai, Nepal”. Int J Appl Sci Biotechnol. 2(3): 328-335. 2014.

[13] ACIAR, "Guidelines for nutrient omission plot studies for rabimaize in 2009-2010". Bangladesh Version for ACIAR Rice Maize and CSISA Projects. 2009.

[14] Paudel, P. and Matsuoka, A, "Factors Influencing Adoption of Improved maize varieties in Nepal: A case study of Chitwan 
district”. Australian Journal of Basic and Applied Sciences. 2(4):823-834. 2008.

[15] Dahal, S, Growth, yield and economics of spring maize (Zea mays L.) in relation to tillage, residue, fertilizer and weed management in Chitwan, Nepal. M.Sc. Ag. Thesis, Tribhuvan University, Department of Agronomy, IAAS, Rampur, Chitwan, Nepal. 2014.

[16] Shrivastav, N, Weed dynamics and productivity of spring maize under different tillage and weed management methods. M.Sc. Ag. Thesis, Tribhuvan University, Department of Agronomy, IAAS, Rampur, Chitwan, Nepal, 2014.

[17] Anonymous, "Sweet Corn". Ohio Vegetable Production Guide. 265-269. 2010

[18] Streit, B, Impact of tillage system, crop sequence, and weed control on the development of weed populations and the degradation of herbicides. Ph. D. Dissertation, Swiss Federal Institute of Technology, Department of Natural Sciences, Zurich, 43p. 2001.

[19] Joshi, K.R, "Effect of time of weeding and levels of $\mathrm{N}$ and $\mathrm{P}_{2} \mathrm{O}_{5}$ fertilizers on the grain yield of maize". Nepal Agric. Res. J. 5: 6970. 2004.

[20] Ademiluyi, B.O. and Abegude C. Miss, "Evaluation of herbicide spray programmes and hoeing for weed control in maize (Zea mays L.) in a south western Nigeria location”. Research Journal of Agronomy. 1(2): 38-41. 2007

[21] Kashe, K, Sindel, B, Kristiansen, P. and Jessop, R, "Effect of tillage on weed seed bank and weed flora in maize (Zea mays)". Seventeenth Australian Weed Conference, 26-30 September 2010, New Frontiers in New Zealand.

[22] Rahman, M.A, Khan, M.A.H. and Rahman, M.M, "Mulch induced morpho-physiological adaptation of quality protein maize". Bangladesh J. Agric. Res. 27(3): 329-340. 2002.

[23] Khan, M.A.H. and Parvej, M.R, "Impact of conservation tillage under organic mulches on the reproductive efficacy and yield of quality protein maize”. The Journal of Agricultural Sciences. 5(2): 52-63. 2010.

[24] Hawaldar, S, Effect of herbicides on weed seed bank and productivity of maize (Zea mays L.). M. Sc. Ag. Thesis, University of Agricultural Sciences, Agronomy Department, Dharwd Institute, Dharwad, Karnataka State, India, 2011.

[25] Kayode, J. and Ademiluyi, B, "Effect of tillage methods on weed control and maize performance in Southwestern Nigeria location". Journal of Sustainable Agriculture. 23(3): 39-45. 2004.

[26] Nieto, H.J, Brondo, M.A. and Gonzalez, J.J, “Critical period of the crop growth cycle for competition for weeds”. Pest Artic. C. 14: 159-166. 1968. 